\title{
Clinical efficacy and safety evaluation of pemetrexed combined with radiotherapy in treatment of patients with lung adenocarcinoma brain metastasis
}

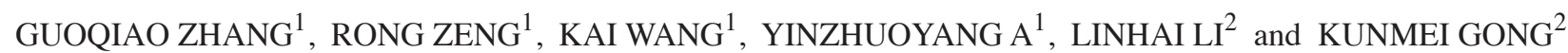 \\ Departments of ${ }^{1}$ Oncology and ${ }^{2}$ General Surgery, The First People's Hospital \\ of Yunnan Province, Kunming, Yunnan 650032, P.R. China
}

Received September 11, 2018; Accepted December 18, 2018

DOI: $10.3892 / \mathrm{ol} .2019 .9894$

\begin{abstract}
Clinical efficacy and adverse reactions of pemetrexed combined with stereotactic gamma-ray radiotherapy in the treatment of patients with lung adenocarcinoma brain metastasis in The First People's Hospital of Yunnan Province were evaluated. A total of 67 patients with lung adenocarcinoma brain metastasis in experimental group were treated with simple pemetrexed chemotherapy, and then with radiotherapy, followed by pemetrexed chemotherapy. Their treatment results were compared with those of 53 patients treated with simple gamma knife in control group. The results were analyzed by comparing the clinical efficacy, side reactions, serum level changes, and survival between the two groups. Among 67 patients in the experimental group, there were 16 cases of complete response (CR), 39 cases of partial response (PR), 7 cases of stable disease (SD) and 5 cases of progressive disease (PD), with an effective rate of $82.09 \%$ (55/67) and a tumor local control rate of $92.54 \%(62 / 67)$. Among 53 patients in the control group, there were 13 cases of CR, 20 cases of PR, 9 cases of SD and 11 cases of PD, with an effective rate of $62.26 \%$ (33/53) and a tumor local control rate of $79.25 \%(42 / 53)$. There were statistically significant differences in the effective rate and local control rate between the two groups $(\mathrm{P}<0.05)$. The 6-, 12- and 24-month survival rates in experimental group were higher than those in control group $(\mathrm{P}<0.05)$. The main adverse reactions after pemetrexed combined with radiotherapy were lower than those after simple radiotherapy $(\mathrm{P}<0.05)$. The expression levels of the tumor markers carcinoembryonic antigen (CEA) and cytokeratin fragment antigen 21-1 (CYFRA21-1) in the two groups of patients after treatment were lower than those before treatment $(\mathrm{P}<0.05)$. After treatment, the expression levels of serum CEA and CYFRA21-1 in the experimental group were significantly lower
\end{abstract}

Correspondence to: Dr Kunmei Gong, Department of General Surgery, The First People's Hospital of Yunnan Province, 157 Jinbi Road, Kunming, Yunnan 650032, P.R. China

E-mail:k7yqzz@163.com; zgqnhm@sina.com

Key words: pemetrexed, gamma knife radiotherapy, combination treatment, lung adenocarcinoma brain metastasis, efficacy than those in the control group $(\mathrm{P}<0.05)$. Pemetrexed combined with radiotherapy in the treatment of lung adenocarcinoma brain metastasis is more effective than simple radiotherapy, with lighter adverse reactions, worthy of clinical application and promotion.

\section{Introduction}

Having a high incidence worldwide (1), lung adenocarcinoma brain metastasis is one of the most common distant metastases. The occurrence of brain metastasis is an important cause of treatment failure and death in patients (2). Patients with lung adenocarcinoma brain metastasis have poor prognoses. The median survival time of untreated patients with brain metastasis is 1 to 2 months, with only $\sim 6$ months in treated patients (3). In recent years, radiotherapy technology has been developed at a high speed, and systemic treatment has become increasingly standardized. In particular, small molecule targeted drugs and chemotherapeutic drugs are widely used in clinical practice. Therefore, the prognoses of patients with lung adenocarcinoma brain metastasis have greatly improved $(4,5)$.

Studies have shown that, compared with simple radiotherapy, targeted or chemotherapeutic drug treatment combined with radiotherapy can have a better synergistic effect and improve the control rate of brain metastasis, prolonging the survival time of patients (6). Having prolonged the survival time of patients with lung cancer brain metastasis to a certain degree, the comprehensive application of treatment methods has significantly improved their quality of life (7). In recent years, extensive attention has been paid by researchers and scholars to stereotactic radiotherapy, especially gamma knife radiotherapy that is minimally invasive and highly integrated, which can avoid the expansion of radiation damage (8). Related studies have proven that (9-11) gamma knife has better efficacy and longer survival time in the treatment of lung cancer brain metastasis tumor than conventional whole brain radiotherapy. Pemetrexed is the first-line medication for lung adenocarcinoma brain metastasis chemotherapy (12). As a new generation of folic acid antagonist, it blocks thymidylate synthase (TS), glycinamide nucleotide transferase (GARFT) and dihydrofolate reductase (DH-FR), further affecting the DNA and RNA syntheses of tumor cells, so as to inhibit their growth and treat tumors (13). 
Clinical practice has shown that the expression level of tumor serum markers in vivo is important for the diagnosis and efficacy judgment of lung adenocarcinoma tumors (14). The comprehensive detection of multiple tumor markers can improve the accuracy rate of lung cancer diagnosis (15). Related studies have shown that the use of multiple lung adenocarcinoma tumor markers for detection can improve the early diagnostic rate of lung cancer patients, providing reliable data support for subsequent efficacy judgment and treatment (16). In this study, cytokeratin fragment antigen 21-1 (CYFRA21-1) and carcinoembryonic antigen (CEA) were selected as markers of lung adenocarcinoma brain metastasis. CEA was first discovered in colon cancer. Subsequent studies have shown that it is highly expressed in epithelial-derived tumors such as lung cancer and esophagus cancer (17). It also plays a very important role in tumor growth and metastasis (18). CYFRA21-1, an acidic protein that constitutes cells, is mainly found in patients with lung cancer and breast cancer. Rare in normal subjects, it is mainly released from the cancerous epithelium and then enters blood circulation (19). A large number of studies have shown that, as the most valuable serum tumor marker in patients with lung cancer brain metastasis, CYFRA21-1 has higher clinical value in the diagnosis, disease monitoring and efficacy judgment of non-small cell lung cancer (20).

The efficacy of pemetrexed combined with stereotactic gamma-ray radiotherapy in the treatment of 67 patients with lung adenocarcinoma brain metastasis was compared with that of simple gamma knife radiotherapy in the traetment of 53 patients, to investigate the clinical significance and safety of pemetrexed combined with radiotherapy in the treatment of lung adenocarcinoma brain metastasis.

\section{Patients and methods}

Research subjects. A retrospective analysis method was used to analyze the cases of 67 patients with lung adenocarcinoma brain metastasis, who were treated with pemetrexed combined with radiotherapy in The First People's Hospital of Yunnan Province (Kunming, China) from May 2013 to December 2015. They were used as experimental group, including 36 males and 31 females, aged 32-78 years, with an average age of $57.48 \pm 16.12$ years, a follow-up time of 1-24 months and an average follow-up time of $15.1 \pm 6.24$ months. At the same time, 53 patients (control group) with lung adenocarcinoma brain metastasis treated with simple gamma knife radiotherapy were selected, including 30 males and 23 females, aged $30-76$ years, with an average age of $55.73 \pm 15.83$ years and an average follow-up time of $14.8 \pm 6.12$ months.

Inclusion criteria: i) patients diagnosed with lung adenocarcinoma brain metastasis by pathology, and with adenocarcinoma by pathological examination of brain metastasis or primary lesions; ii) patients with normal electrocardiogram, urine routine, liver and kidney function, coagulation function, blood pressure and blood routine before treatment; iii) patients with no history of operation and trauma within 4 weeks; iv) patients with no hemoptysis, epistaxis or other kind of bleeding; v) patients with no severe vascular invasion observed in imaging examination results; and vi) patients with survival time estimated to be $\geq 3$ months.
Exclusion criteria: i) patients who refused operation or who could not have operation; ii) patients with other cancers; iii) patients with liver dysfunction; iv) those with autoimmune system defects; v) long-term smokers; vi) pregnant or lactating women; and vii) patients receiving any other anti-tumor treatment than brain metastasis radiotherapy during treatment.

The study was approved by the Ethics Committee of The First People's Hospital of Yunnan Province. Patients who participated in this research, signed an informed consent.

\section{Experimental methods}

Treatment plan. Control group: gamma knife was used in the treatment. Local anesthesia was performed on the patients prior to the installation of Leksell stereotactic stent (Elekta Instrument AB, Stockholm, Sweden). A 1.5-T magnetic resonance imaging (MRI) system (MAGNETOM Avanto; Siemens AG, Munich, Germany) was used to perform non-interval and enhanced scans on the thin layer $(3 \mathrm{~mm})$ of the lesion area. The obtained high-resolution image was transmitted to the treatment planning system (TPS) in a private network for three-dimensional image reconstruction display and stereoscopic positioning of the target area. The tumor was encased with a $50 \%$ isodose curve, with a peripheral dose of 22-26 Gy and an average of 18.5 Gy for treatment, and the stereotactic frame was removed after the treatment. Experimental group: A total of $500 \mathrm{mg} / \mathrm{m}^{2}$ pemetrexed (SFDA approval no. H20080177, 500 mg/branch; Nanjing Pharmaceutical Co., Ltd., Nanjing, China) was added to $100 \mathrm{ml}$ of saline for dissolution. After pemetrexed treatment given for 2 cycles, stereotactic gamma-ray radiation system (OUR New Med., Shenzhen, China) was used. In order to minimize the difference between source skin distance and source tumor distance, CT scans were performed in the prone or supine position. The image was input to the WBS-TPS workstation to confirm the clinical target volume and adjust the exposure field. According to the size of the lesions, $50-70 \%$ isodose curve was used to enclose the clinical target area. The optimization was conducted according to the probability of complications and tumor control in normal tissues. The isodose curve was $60-70 \%$ for wrapping, the peripheral dose was 5-7 Gy/time, and the total dose was 40-56 Gy. The operation was the same as gamma knife treatment in control group. Pemetrexed chemotherapy for 2 cycles was performed after radiotherapy.

Altogether 21 days were considered to be 1 treatment cycle for both groups. The two groups of patients received symptomatic supportive treatments such as glucocorticoids and mannitol dehydration, intracranial pressure before and after gamma knife treatment. Experimental group was given folic acid (SFDA approval no. H32023302, $400 \mu \mathrm{g} /$ tablet; Changzhou Pharmaceutical Factory Co., Ltd., Changzhou, China) on the 7th day before chemotherapy, $400 \mu \mathrm{g} /$ time, orally administered once/day, until the 21st day after chemotherapy. Vitamin B12 (SFDA approval no. H32021841, $500 \mu \mathrm{g} / \mathrm{branch}$; Jinling Pharmaceutical Co., Ltd., Nanjing, China) was supplemented 1,000 $\mu \mathrm{g} /$ time, intramuscularly injected, with injection once every 4 chemotherapy cycles. Dexamethasone acetate tablet (SFDA approval no. H22023498, 0.75 mg/tablet; Changchun Dirui Pharmaceutical Co., Ltd., Changchun, China) was orally administered once daily, $7.5 \mathrm{mg} /$ time before the 1st day of pemetrexed, on the day and on the 2nd day 
Table I. Comparison of clinical basic conditions between two groups of patients with lung adenocarcinoma brain metastasis $[\mathrm{n}(\%)]$.

\begin{tabular}{|c|c|c|c|c|}
\hline Clinical characteristics & $\begin{array}{l}\text { Experimental group } \\
\qquad(\mathrm{n}=67)\end{array}$ & $\begin{array}{l}\text { Control group } \\
\quad(\mathrm{n}=53)\end{array}$ & $\chi^{2}$ & P-value \\
\hline Sex & & & 0.099 & 0.753 \\
\hline Male & $36(53.73)$ & $30(56.60)$ & & \\
\hline Female & $31(46.27)$ & $23(43.40)$ & & \\
\hline Age (years) & & & 0.203 & 0.653 \\
\hline$\leq 65$ & $39(58.21)$ & $33(62.26)$ & & \\
\hline$>65$ & $28(41.79)$ & $20(37.74)$ & & \\
\hline \multicolumn{5}{|l|}{ Smoking } \\
\hline Yes & $12(17.91)$ & $10(18.87)$ & 0.018 & 0.893 \\
\hline No & $55(82.09)$ & $43(81.13)$ & & \\
\hline Number of brain metastasis lesions (1-10 lesions) & & & 1.315 & 0.251 \\
\hline$\leq 3$ & $40(59.70)$ & $37(69.81)$ & & \\
\hline$>3$ & $27(40.30)$ & $16(30.19)$ & & \\
\hline Maximum diameter of brain metastasis lesion $(\mathrm{cm})$ & & & 1.079 & 0.299 \\
\hline$\leq 3$ & $38(56.72)$ & $35(66.04)$ & & \\
\hline$>3$ & $29(43.28)$ & $18(33.96)$ & & \\
\hline Primary tumor number & & & 0.056 & 0.813 \\
\hline Simple & $48(71.64)$ & $39(73.58)$ & & \\
\hline Multiple & $19(28.36)$ & $14(26.42)$ & & \\
\hline Primary tumor site & & & 2.033 & 0.154 \\
\hline Peripheral type & $44(65.67)$ & $28(52.83)$ & & \\
\hline Central type & $23(34.33)$ & $25(47.17)$ & & \\
\hline ECOG score & & & 0.007 & 0.934 \\
\hline$\leq 2$ & $51(76.12)$ & $40(75.47)$ & & \\
\hline$>2$ & $16(23.88)$ & $13(24.53)$ & & \\
\hline
\end{tabular}

of medication. Blood routine, liver and kidney function and electrolyte examinations were required weekly during treatment. The clinical efficacy and adverse reactions in the pemetrexed combined with radiotherapy group and simple gamma knife group were observed and recorded.

CEA and CYFRA21-1 determination. In the morning, $3 \mathrm{ml}$ of fasting venous blood were collected from all subjects with a vacuum blood lancet, before and after treatment, and were placed in a centrifuge. Centrifugation was performed at $3,000 \mathrm{x}$ g for $15 \mathrm{~min}$ at $4^{\circ} \mathrm{C}$. The slurry in the test tube was then carefully aspirated to obtain serum. The instruments used were Roche Cobas E601 electrochemical luminescence immunoassay analyzer (Roche Diagnostics Co., Ltd., Shanghai, China), CEA detection kit (xy-302; Shanghai Xinyu Biotechnology Co., Ltd., Shanghai, China) and CYFRA21-1 (JK-EA00523; Shanghai Jingkang Bioengineering Co., Shanghai, China). Electrochemiluminescence was used to detect serum CEA and CYFRA21-1 levels in the two groups.

Follow-up and observation indicators. After treatment, patients in the two groups were followed up by visiting the hospital and by telephone. Their survival times and diameter rates of survival lesion were recorded at 6th, 12th, and 24th months after treatment. Therapeutic effects were evaluated according to the evaluation criteria in solid tumors of the World Health
Organization (WHO), and the overall efficacy was recorded. If the disease mutated, the patient was promptly treated. The magnetic resonance examination was strengthened at each time of follow-up in clinic. Survival time was recorded from the 1st day of patients with lung adenocarcinoma brain metastasis treated with gamma knife to the death or follow-up deadline. The last follow-up time of this study was December 23, 2017.

Response evaluation criteria (21). The evaluation was based on the Response Evaluation Criteria in Solid Tumors (RECIST). Complete response (CR): all lesions disappear and this is maintained for 4 weeks. Partial response (PR): the total lesion diameter decreases by $\geq 30 \%$ and maintained for 4 weeks. Progressive disease (PD): the total lesion diameter increases by $\geq 20 \%$ or new lesions occur. Stable disease (SD): the total lesion diameter decreases but does not reach $\mathrm{PR}$, or increases but does not reach $\mathrm{PD}$. The effective rate $=(\mathrm{CR}+\mathrm{PR}) /$ total number of cases $\times 100 \%$. The tumor local control rate $=(\mathrm{CR}+\mathrm{PR}+\mathrm{SD}) /$ total number of cases $\times 100 \%$.

Determination of toxic and side effects. Following the common chemotherapeutic drugs grading criteria of the WHO (22), the adverse reactions observed in patients with lung adenocarcinoma brain metastasis, such as nausea and vomiting, fatigue and myelosuppression caused by drug use were graded. Then, the incidence of toxic and side effects 
Table II. Comparison of clinical efficacy between two groups of patients [n (\%)].

\begin{tabular}{|c|c|c|c|c|c|c|c|}
\hline Group & $\mathrm{n}$ & $\mathrm{CR}$ & PR & SD & PD & $\begin{array}{l}\text { Effective } \\
\text { rate }\end{array}$ & $\begin{array}{l}\text { Tumor loca } \\
\text { control rate }\end{array}$ \\
\hline Experimental group & 67 & $16(23.88)$ & $39(58.21)$ & $7(10.45)$ & $5(7.46)$ & $82.09 \%$ & $92.54 \%$ \\
\hline Control group & 53 & $13(24.53)$ & $20(37.74)$ & $9(16.98)$ & $11(20.75)$ & $62.26 \%$ & $79.25 \%$ \\
\hline$\chi^{2}$ & & & & & & 5.948 & 4.524 \\
\hline P-value & & & & & & $<0.05$ & $<0.05$ \\
\hline
\end{tabular}

CR, complete response; PR, partial response; $\mathrm{SD}$, stable disease; $\mathrm{PD}$, progressive disease.

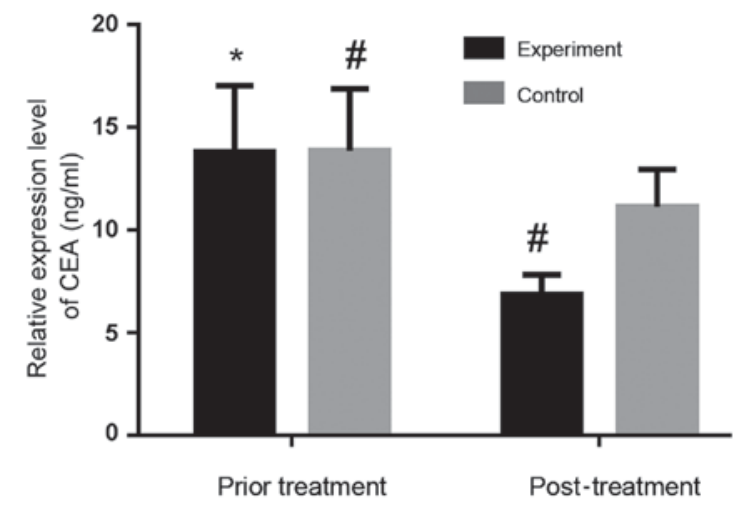

Figure 1. Serum CEA expression level before and after treatment in experimental and control group. The serum CEA level after treatment was significantly lower than that before treatment in experimental group $(\mathrm{P}<0.05)$, as well as in control group $(\mathrm{P}<0.05)$. Serum CEA level after treatment was lower in experimental group than that in control group $(\mathrm{P}<0.05) .{ }^{*} \mathrm{P}<0.05$, compared with experimental group after treatment; ${ }^{\#} \mathrm{P}<0.05$, compared with control group after treatment; CEA, carcinoembryonic antigen.

was compared between the pemetrexed combined with radiotherapy group and the simple gamma knife group.

Statistical methods. SPSS 17.0 statistical software (SPSS, Inc., Chicago, IL, USA) was used to statistically analyze the experimental data. Enumeration data were expressed as n (\%). The Chi-square test was used for the comparison between groups. Measurement data were expressed as mean \pm standard deviation. Paired t-test was used for data comparison before and after treatment in the group. The paired t-test was used for the comparison in the group before and after treatment. Multiple comparisons were performed using ANOVA followed by the SNK test which was used as the post hoc test. The Kaplan Meier test for the survival analysis and log-rank test were also used. $\mathrm{P}<0.05$ was considered to indicate a statistically significant difference.

\section{Results}

Comparison of clinical basic conditions between two groups of patients with lung adenocarcinoma brain metastasis. The clinical basic conditions of patients were analyzed, as shown in Table I. There was no significant difference between the experimental and control group, in sex, age, smoking, number of brain metastasis lesions, maximum diameter of brain metastasis lesion, primary tumor number, primary tumor site and ECOG score factors in patient behavioral status $(\mathrm{P}>0.05)$.

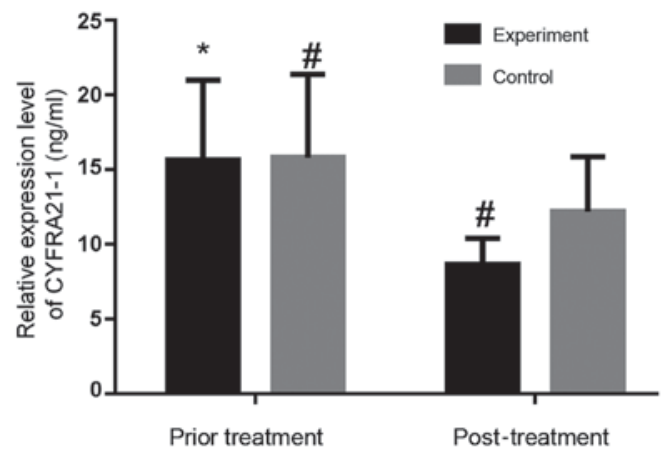

Figure 2. Serum CYFRA21-1 expression level before and after treatment in experimental and control group. The serum CYFRA21-1 level after treatment was significantly lower than that before treatment in experimental group $(\mathrm{P}<0.05)$, as well as in control group $(\mathrm{P}<0.05)$. Serum CYFRA21-1 level after treatment was lower in experimental group than that in control group $(\mathrm{P}<0.05) .{ }^{*} \mathrm{P}<0.05$, compared with experimental group after treatment; ${ }^{\#} \mathrm{P}<0.05$, compared with control group after treatment; CYFRA21-1, cytokeratin fragment antigen 21-1.

Comparison of clinical efficacy between two groups of patients. All treatments and follow-ups were performed on both groups of patients. The treatment effective rate and tumor local control rate of patients were compared between experimental and control group, and were found to be significantly different $(\mathrm{P}<0.05)$. The results showed that the treatment effective rate and tumor local control rate were significantly higher in experimental group than those in control group, and the clinical efficacy was better in experimental group than that in control group (Table II).

Comparison of the serum levels. In experimental group, serum CEA and CYFRA21-1 concentrations before treatment were $13.78 \pm 3.24$ and $15.67 \pm 5.32 \mathrm{ng} / \mathrm{ml}$, respectively. Those after treatment were $6.85 \pm 0.98$ and $8.65 \pm 1.75 \mathrm{ng} / \mathrm{ml}$, respectively. Serum levels of CEA and CYFRA21-1 after treatment were significantly lower than those before treatment in the experimental group $(\mathrm{P}<0.05)$. In control group, the serum CEA and CYFRA21-1 levels before treatment were $13.85 \pm 3.02$ and $15.82 \pm 5.56 \mathrm{ng} / \mathrm{ml}$, respectively. Those after treatment were $11.12 \pm 1.82$ and $11.12 \pm 1.82 \mathrm{ng} / \mathrm{ml}$, respectively. Serum levels after treatment were lower than those before treatment in the control group $(\mathrm{P}=0.0004,0.0033)$. After treatment, the expression of serum CEA and CYFRA21-1 in the experimental group was significantly lower than those in the control group $(\mathrm{P}<0.05)$. Figs. 1 and 2 show that the overall 
Table III. Comparison of side reactions between two groups [n (\%)].

I-II

\begin{tabular}{|c|c|c|c|c|c|c|}
\hline Group & $\mathrm{n}$ & Myelosuppression & $\begin{array}{c}\text { Fatigue } \\
\text { vomiting }\end{array}$ & $\begin{array}{l}\text { Nausea and } \\
\text { esophagitis }\end{array}$ & Radiation & $\begin{array}{c}\text { Radiation } \\
\text { pneumonitis }\end{array}$ \\
\hline Experimental group & 67 & $18(26.87)$ & $39(58.21)$ & $34(50.75)$ & $9(13.43)$ & $4(5.97)$ \\
\hline Control group & 53 & $26(49.06)^{\mathrm{a}}$ & $41(77.36)^{\mathrm{a}}$ & $46(86.79)^{\mathrm{a}}$ & $15(28.30)^{\mathrm{a}}$ & $10(18.87)^{\mathrm{a}}$ \\
\hline$\chi^{2}$ & & 6.28 & 4.88 & 17.30 & 4.09 & 4.78 \\
\hline P-value & & 0.012 & 0.027 & $<0.001$ & 0.043 & 0.029 \\
\hline
\end{tabular}

${ }^{\mathrm{a}} \mathrm{P}<0.05$, compared with experimental group.

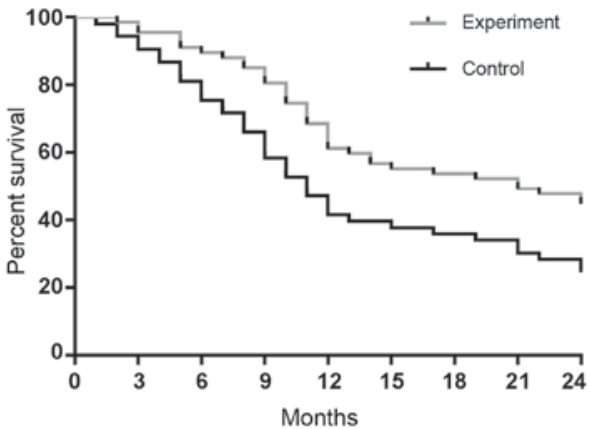

Figure 3. Survival curves of two groups. There were significant differences in the 6-, 12- and 24-month survival rates between the experimental and control group $(\mathrm{P}<0.05)$.

decrease is more obvious in the experimental group than that in the control group.

Comparison of side reactions between two groups. Both groups had better tolerance and fewer adverse reactions in grades III-IV. Severe side reactions in grades I-II were compared between experimental and control groups. The proportion of toxic and side effects was significantly lower in experimental group than that in control group, and there was a significant difference in the incidence of toxic and side effects between the two groups $(\mathrm{P}<0.05$; Table III).

Comparison of survival analysis between two groups. The 6-, 12- and 24-month survival rates in the experimental group were $89.55 \%$ (60/67), 61.19\% (41/67) and $44.78 \%(30 / 67)$, respectively, with a median survival time of 13.1 months. Those in control group were $75.47 \%$ (40/53), 41.51\% (22/53) and $24.53 \%(13 / 53)$, respectively, with a median survival time of 10.8 months. The survival rate and median survival time were higher in experimental group than those in control group, and there were significant differences in the 6-, 12- and 24-month survival rates between the two groups $(\mathrm{P}<0.05)$. Survival curves were plotted according to the survival data of experimental and control groups (Fig. 3).

\section{Discussion}

Lung adenocarcinoma is one of the most common malignant tumors in respiratory diseases, and the incidence of brain metastasis is lower than that of other types of metastases. Nevertheless, once occurring, it causes serious consequences, which is also an important reason for poor prognosis and death in patients with advanced lung adenocarcinoma (2). In clinic, the use of a simple treatment method is not ideal. Gamma knife radiotherapy is often used in patients with lung adenocarcinoma brain metastasis, mainly because its application can improve the poor positioning of whole brain radiotherapy and the shortage of radiation dose to some extent (23). In addition, gamma knife has the advantages of no craniotomy and small damage. The damage degree of the tissue near the target is significantly reduced, with the edge of the lesion after operation being sharper (24). Pemetrexed is the first-line of chemotherapy for lung adenocarcinoma (12). It has a stronger ability of blood brain barrier penetration. Pemetrexed has multiple targets, so it shows broad-spectrum anti-tumor effect in clinical application, with exact efficacy (25).

Serum CEA is one of the most widely used tumor markers in clinical practice, and changes in CEA level have a certain judgment value for predicting the therapeutic efficacy and prognoses of patients with lung adenocarcinoma brain metastasis (26). CYFRA21-1, having higher sensitivity and specificity for lung cancer brain metastasis than CEA, is the preferred tumor marker (27). Commonly used to monitor lung adenocarcinoma brain metastasis, the expression levels of serum CEA and CYFRA21-1 in patients are often considered to be positively correlated with tumor recurrence and metastasis, but negatively correlated with the survival rate of lung adenocarcinoma patients (28). In this study, the expression levels of both kinds of serum before treatment were higher than those after treatment in experimental and control group. The serum levels after treatment were significantly lower than those before treatment in the experimental group $(\mathrm{P}<0.05)$, as well as in the control group $(\mathrm{P}<0.05)$. Additionally, the decrease of serum CEA and CYFRA21-1 levels after treatment was more obvious in experimental group than that in control group. The detection of CEA and CYFRA21-1 indicates that pemetrexed combined with radiotherapy is more effective than simple gamma knife treatment. Therefore, the detection of tumor marker content in serum has certain guiding significance in judging the efficacy of drug treatment (29).

In this study, there was no significant difference between experimental and control group, in sex, age, smoking, number of brain metastasis lesions, maximum diameter of brain 
metastasis lesion, primary tumor number, primary tumor site and ECOG score factors in patient behavioral status ( $\mathrm{P}>0.05)$. It was found that the effective rate and local control rate in the experimental group were significantly higher than those in the control group. The differences between the groups were statistically significant $(\mathrm{P}<0.05)$, suggesting that this combination regimen can improve patients' quality of life to some extent. This is mainly due to the fact that pemetrexed can reduce the incidence of lung adenocarcinoma brain metastasis (12). By regulating multiple enzymes in the folate-dependent metabolic pathway, pemetrexed mainly acts on multiple targets in the body to act as an anti-folate, further effectively inhibiting the synthesis and metabolism of tumor cells in vivo $(30,31)$. The present study showed that the incidence of adverse reactions such as toxic and side effects was lower in the experimental group than that in the control group, with a statistically significant difference $(\mathrm{P}<0.05)$. This indicates that the safety of the combination treatment is higher than that of the simple radiotherapy. The survival time was compared between the experimental and control group. The results showed that the median survival time of patients was longer in the experimental group than that in the control group, and there were significant differences in the 6-, 12- and 24-month survival rates of patients between the two groups $(\mathrm{P}<0.05)$. This suggests that pemetrexed combined with radiotherapy can prolong the survival time of lung adenocarcinoma patients to some extent. The study of Tiseo et al (32) has shown that pemetrexed-disodium combined with gamma knife in the treatment of non-small cell lung cancer brain metastasis has better clinical efficacy and safety than simple gamma knife treatment, with certain clinical promotion and application value. This is consistent with the findings of our study.

The clinical significance and safety evaluation of pemetrexed combined with radiotherapy were explored by efficacy evaluation, tumor marker detection, side reaction judgment, and survival analysis. The investigation and analysis are more comprehensive, so the results are convincing, conducive to providing reference for the clinic. However, in the screening of lung adenocarcinoma patients and collection of relevant data, subjective factors are inevitable. Also, there is a limitation in the design. Four further experimental groups could be included in future experiments to validate the data: i) simple gamma-knife only, ii) whole body gamma knife only, iii) pemetrexed only, and iv) a combination of pemetrexed and whole body gamma knife. In addition, in subsequent studies, the staging of lung adenocarcinoma brain metastasis should be studied, to compare the efficacy difference between pemetrexed combined with radiotherapy and simple gamma knife and the incidence of adverse reactions in the staging.

In summary, pemetrexed combined with stereotactic gamma-ray radiotherapy in the treatment of patients with lung adenocarcinoma brain metastasis is more effective, with lighter adverse reactions and higher safety. Having certain clinical value, it can provide reference for clinically selecting treatment methods of lung adenocarcinoma brain metastasis.

\section{Acknowledgements}

Not applicable.

\section{Funding}

This study was supported by the Yunnan Province Health Science and Technology Project (no. 2014NS266).

\section{Availability of data and materials}

The datasets used and/or analyzed during the current study are available from the corresponding author on reasonable request.

\section{Authors' contributions}

GZ and RZ assisted with CEA and CYFRA21-1 determination. $\mathrm{KW}$ and YA collected and analyzed the general information of patients. LL and KG were responsible for the surgery. All authors read and approved the final manuscript.

\section{Ethics approval and consent to participate}

This study was approved by the Ethics Committee of The First People's Hospital of Yunnan Province (Kunming, China). Patients who participated in this research, signed an informed consent and had complete clinical data.

\section{Patient consent for publication}

Not applicable.

\section{Competing interests}

The authors declare that they have no competing interests.

\section{References}

1. Ning M, Chunhua M, Rong J, Yuan L, Jinduo L, Bin W and Liwei S: Diagnostic value of circulating tumor cells in cerebrospinal fluid. Open Med (Wars) 11: 21-24, 2016.

2. Kawabe T, Phi JH, Yamamoto M, Kim DG, Barfod BE and Urakawa Y: Treatment of brain metastasis from lung cancer. Prog Neurol Surg 25: 148-155, 2012.

3. Fidler IJ: The biology of brain metastasis: challenges for therapy. Cancer J 21: 284-293, 2015

4. Johung KL, Yeh N, Desai NB, Williams TM, Lautenschlaeger T, Arvold ND, Ning MS, Attia A, Lovly CM, Goldberg S, et al: Extended survival and prognostic factors for patients with ALK-rearranged non-small-cell lung cancer and brain metastasis. J Clin Oncol 34: 123-129, 2016.

5. Jamal-Hanjani M and Spicer J: Epidermal growth factor receptor tyrosine kinase inhibitors in the treatment of epidermal growth factor receptor-mutant non-small cell lung cancer metastatic to the brain. Clin Cancer Res 18: 938-944, 2012.

6. Liu WJ, Zeng XT, Qin HF, Gao HJ, Bi WJ and Liu XQ: Whole brain radiotherapy plus chemotherapy in the treatment of brain metastases from lung cancer: A meta-analysis of 19 randomized controlled trails. Asian Pac J Cancer Prev 13: 3253-3258, 2012.

7. Shi Y, Sun Y, Yu J, Ding C, Ma Z, Wang Z, Wang D, Wang Z, Wang M, Wang Y, et al: China experts consensus on the diagnosis and treatment of brain metastases of lung cancer (2017 version). Zhongguo Fei Ai Za Zhi 20: 1-13, 2017 (In Chinese).

8. Rahn DA III, Ray DK, Schlesinger DJ, Steiner L, Sheehan JP, O'Quigley JM and Rich T: Gamma knife radiosurgery for brain metastasis of nonsmall cell lung cancer: Is there a difference in outcome between morning and afternoon treatment? Cancer 117: 414-420, 2011.

9. Peacock KH and Lesser GJ: Current therapeutic approaches in patients with brain metastases. Curr Treat Options Oncol 7: 479-489, 2006. 
10. Pan HC, Sheehan J, Stroila M, Steiner M and Steiner L: Gamma knife surgery for brain metastases from lung cancer. J Neurosurg 102 (Suppl): 128-133, 2005.

11. Gerosa M, Nicolato A, Foroni R, Tomazzoli L and Bricolo A: Analysis of long-term outcomes and prognostic factors in patients with non-small cell lung cancer brain metastases treated by gamma knife radiosurgery. J Neurosurg 102 (Suppl): 75-80, 2005.

12. Ortuzar W, Hanna N, Pennella E, Peng G, Langer C, Monberg M and Scagliotti G: Brain metastases as the primary site of relapse in two randomized phase III pemetrexed trials in advanced non-small-cell lung cancer. Clin Lung Cancer 13: 24-30, 2012.

13. Shimizu T, Nakanishi Y, Nakagawa Y, Tsujino I, Takahashi N, Nemoto $\mathrm{N}$ and Hashimoto S: Association between expression of thymidylate synthase, dihydrofolate reductase, and glycinamide ribonucleotide formyltransferase and efficacy of pemetrexed in advanced non-small cell lung cancer. Anticancer Res 32: 4589-4596, 2012.

14. Siegel RL, Miller KD and Jemal A: Cancer statistics, 2016. CA Cancer J Clin 66: 7-30, 2016.

15. Shi Y, Sun Y, Ding C, Wang Z, Wang C, Bai C, Bai C, Feng J, Liu X, $\mathrm{Li} \mathrm{F}$, et al: China experts consensus on icotinib for non-small cell lung cancer treatment (2016 version). Zhongguo Fei Ai Za Zhi 19: 489-494, 2016 (In Chinese)

16. Wang XF, Wu YH, Wang MS and Wang YS: CEA, AFP, CA125, CA153 and CA199 in malignant pleural effusions predict the cause. Asian Pac J Cancer Prev 15: 363-368, 2014.

17. Nagashima T, Sakao Y, Mun M, Ishikawa Y, Nakagawa K, Masuda M and Okumura S: A clinicopathological study of resected small-sized squamous cell carcinomas of the peripheral lung: Prognostic significance of serum carcinoembryonic antigen levels. Ann Thorac Cardiovasc Surg 19: 351-357, 2013.

18. Kulpa J, Wójcik E, Reinfuss M and Kołodziejski L: Carcinoembryonic antigen, squamous cell carcinoma antigen, CYFRA 21-1, and neuron-specific enolase in squamous cell lung cancer patients. Clin Chem 48: 1931-1937, 2002.

19. Sertić Milić H, Franjević A, Bubanović G, Marušić A, Nikolić I and Puljić I: Size, edge, and stage of NSCLC determine the release of CYFRA 21-1 in bloodstream. Wien Klin Wochenschr 127: 465-471, 2015.

20. Szturmowicz M, Rudziński P, Kacprzak A, Langfort R, Bestry I, Broniarek-Samson B and Orłowski T: Prognostic value of serum C-reactive protein (CRP) and cytokeratin 19 fragments (Cyfra 21-1) but not carcinoembryonic antigen (CEA) in surgically treated patients with non-small cell lung cancer. Pneumonol Alergol Pol 82: 422-429, 2014.

21. Ramalingam S and Belani C: Systemic chemotherapy for advanced non-small cell lung cancer: recent advances and future directions. Oncologist 13 (Suppl 1): 5-13, 2008.

22. Muallaoglu S, Disel U, Mertsoylu H, Besen A, Karadeniz C, Taner Sumbul A, Abali H and Ozyilkan O: Acute infusion reactions to chemotherapeutic drugs: a single institute experience. J BUON 18: 261-267, 2013.
23. Lindvall P, Bergström P, Löfroth PO, Henriksson R and Bergenheim AT: Hypofractionated conformal stereotactic radiotherapy alone or in combination with whole-brain radiotherapy in patients with cerebral metastases. Int J Radiat Oncol Biol Phys 61: 1460-1466, 2005.

24. Park SJ, Kim HT, Lee DH, Kim KP, Kim SW, Suh C and Lee JS: Efficacy of epidermal growth factor receptor tyrosine kinase inhibitors for brain metastasis in non-small cell lung cancer patients harboring either exon 19 or 21 mutation. Lung Cancer 77: 556-560, 2012.

25. Al-Saleh K, Quinton C and Ellis PM: Role of pemetrexed in advanced non-small-cell lung cancer: meta-analysis of randomized controlled trials, with histology subgroup analysis. Curr Oncol 19: e9-e15, 2012.

26. Arrieta O, Villarreal-Garza C, Martínez-Barrera L, Morales M, Dorantes-Gallareta Y, Peña-Curiel O, Contreras-Reyes S, Macedo-Pérez EO and Alatorre-Alexander J: Usefulness of serum carcinoembryonic antigen (CEA) in evaluating response to chemotherapy in patients with advanced non small-cell lung cancer: a prospective cohort study. BMC Cancer 13: 254, 2013.

27. Hoang T, Xu R, Schiller JH, Bonomi P and Johnson DH: Clinical model to predict survival in chemonaive patients with advanced non-small-cell lung cancer treated with third-generation chemotherapy regimens based on eastern cooperative oncology group data. J Clin Oncol 23: 175-183, 2005.

28. Muley T, Fetz TH, Dienemann H, Hoffmann H, Herth FJ, Meister M and Ebert W: Tumor volume and tumor marker index based on CYFRA 21-1 and CEA are strong prognostic factors in operated early stage NSCLC. Lung Cancer 60: 408-415, 2008.

29. Guo J, Yu J, Song X and Mi H: Serum CA125, CA199 and CEA combined detection for epithelial ovarian cancer diagnosis: A meta-analysis. Open Med (Wars) 12: 131-137, 2017.

30. Zhao R, Qiu A, Tsai E, Jansen M, Akabas MH and Goldman ID: The proton-coupled folate transporter: Impact on pemetrexed transport and on antifolates activities compared with the reduced folate carrier. Mol Pharmacol 74: 854-862, 2008.

31. Chattopadhyay S, Moran RG and Goldman ID: Pemetrexed: biochemical and cellular pharmacology, mechanisms, and clinical applications. Mol Cancer Ther 6: 404-417, 2007.

32. Tiseo M, Giovannetti E, Tibaldi C, Camerini A, Di Costanzo F, Barbieri F, Burgers JA, Vincent A, Peters GJ, Smit EF and Ardizzoni A: Pharmacogenetic study of patients with advanced non-small cell lung cancer (NSCLC) treated with second-line pemetrexed or pemetrexed-carboplatin. Lung Cancer 78: 92-99, 2012.

This work is licensed under a Creative Commons Attribution-NonCommercial-NoDerivatives 4.0 International (CC BY-NC-ND 4.0) License. 